\title{
Light Up the Tissue and Brighten Your Patients' Odds
}

\author{
Max J. H. Witjes, MD, DDS, $\mathrm{PhD}^{1}$, Christian S. Betz, MD, $\mathrm{PhD}^{2}$, and Go M. van Dam, MD, $\mathrm{PhD}^{3}$ \\ ${ }^{1}$ Department of Oral and Maxillofacial Surgery, University Medical Center Groningen, University of Groningen, \\ Groningen, The Netherlands; ${ }^{2}$ Department of Otorhinolaryngology, Universitätsklinikum Hamburg-Eppendorf, Hamburg, \\ Germany; ${ }^{3}$ Department of Nuclear Medicine and Molecular Imaging, University Medical Center Groningen, University of \\ Groningen, Groningen, The Netherlands
}

In the past 2 decades, the strategy in advanced laryngeal cancer has shifted from surgery to so-called organ-sparing treatment using radiation or concomitant chemoradiation as the primary treatment modality. ${ }^{1}$ This paradigm shift towards non-surgical treatment has been beneficial for the preservation of swallowing and speech in laryngeal cancer. When (chemo)radiation has failed, salvage total laryngectomy is the only treatment option with curative intent. With the introduction of organ preservation strategies, the number of laryngectomies as primary treatment have declined, while salvage laryngectomies have increased in time. Surgery in radiated tissue can be challenging, with often compromised postoperative wound healing. In head and neck cancer, surgery can lead to postoperative tissue breakdown, with profound effects on functions such as speech and swallowing with an increased risk of aspiration. In salvage laryngectomy, the complication rates are much higher than in primary laryngectomy. Possible complications of a total laryngectomy after (chemo)radiation include issues such as pharyngocutaneous fistula, tracheoesophageal fistula, wound infection, pharyngeal stenosis/strictures, bleeding, and a higher incidence of free vascularized flap loss. The incidence of overall postoperative complications after salvage laryngectomy is high and has been reported to be over $60 \%$. $^{2}$ These complications result in prolonged hospital stay and re-interventions after salvage laryngectomy, with a burden for the patient and increased costs of health care.

(C) Society of Surgical Oncology 2019

First Received: 19 February 2019;

Published Online: 7 March 2019

M. J. H. Witjes, MD, DDS, PhD

e-mail: m.j.h.witjes@umcg.nl
The study by Hoesli et al. entitled "Intraoperative fluorescent angiography predicts pharyngocutaneous fistula after salvage laryngectomy" addresses the issue of predicting postoperative tissue breakdown after salvage total laryngectomy. ${ }^{3}$ Predicting durante operationem which patients may suffer from a pharyngocutaneous fistula postoperatively has the potential to change the strategy in the operating room. Using a straightforward approach of intraoperative laser angiography and indocyanine green (ICG) as the tracer, the authors used this real-time intraoperative tool for studying the vascularization of the tissues after laryngectomy. The trial was set up as a prospective trial in 41 patients who underwent salvage laryngectomy, with the primary outcome being the presence of a pharyngocutaneous fistula within 30 days after surgery versus the fluorescence curve (peak fluorescence signal/time to peak signal) of the pharyngeal tissues immediately after removal of the larynx. From the receiver operating characteristic (ROC) curve, the authors were able to calculate a cut-off value that could identify the risk of developing pharyngocutaneous fistulas between the highrisk and low-risk groups. The authors suggest that the tool could potentially justify removal of the ICG-dark, hypoperfused pharyngeal tissues in order to reduce the incidence of postoperative pharyngocutaneous fistulas.

The paper from Hoesli and colleagues is one in a long line of papers demonstrating the benefit of using ICG for identifying hypoperfused or leaky tissue. ICG was first introduced in medicine in the mid-1950s. It binds to intravascular albumin and is rapidly cleared from the tissue in $<20 \mathrm{~min}$, with little risk of adverse effects for patients. It has been studied in several medical fields and has US FDA approval of angiography in ophthalmology, cardiology and neurosurgery, and is allowed to be used with certified imaging systems such as intraoperative cameras 
and microscopes. ${ }^{4}$ Off-label use of ICG is documented in abdominal surgery, microvascular surgery (free vascularized tissue flaps), and oncologic staging (sentinel node identification). The fluorescence signal is in the near-infrared region $(>750 \mathrm{~nm})$ that separates the signal from background fluorescence, since native endogenous tissue fluorescence is excited at much lower wavelength regions. ICG has been used in experimental settings mainly due to the lack of available systems. With the introduction of easy-to-use near-infrared cameras for ICG detection, a greater number of institutes are using the technology more routinely.

ICG fluorescence has shown to be beneficial in vessel angiography for the prediction of areas of hypoperfusion, and in high-risk surgical anastomosis. It can serve as a supportive decision-making tool during surgery. Moyer and Losken showed if there was $<25 \%$ ICG perfusion, non-viable skin occurred in $90 \%$ of cases in patients who underwent mastectomy. ${ }^{5}$ In abdominal wall surgery, ICG fluorescence showed hypoperfused areas in panniculectomy or ventral hernia repair. ${ }^{6}$ Similar benefits have been show during reconstructive microsurgery to detect suitable perforator vessels and tissue perfusion after completing the microanastomosis in free vascularized tissue transfer. ${ }^{7}$ In colectomy patients, ICG fluorescence perfusion imaging led to a change in surgical plan in $7.9 \%$ of patients, resulting in a lower anastomotic leak rate $(1.4 \%)$ than the historical rates $(3-15 \%){ }^{8}$

Most study designs in ICG angiography were investigations of small cohorts. Several randomized clinical trials are currently being performed to establish the benefit of ICG angiography. Hoesli et al. have developed a method of intraoperative analysis of the ICG fluorescence curve during salvage laryngectomy. The next step in further research should be the design of a randomized trial with the aim of performing an intervention in which hypoperfused pharyngeal tissue is removed prior to closure, expecting it would lead to a reduced complication rate.

\section{REFERENCES}

1. Forastiere AA, Goepfert H, Maor M, et al. Concurrent chemotherapy and radiotherapy for organ preservation in advanced laryngeal cancer. N Engl J Med. 2003;349(22):2091-8.

2. Hasan Z, Dwivedi RC, Gunaratne DA, et al. Systematic review and meta-analysis of the complications of salvage total laryngectomy. Eur J Surg Oncol. 2017;43(1):42-51.

3. Hoesli R, Brennan JR, Rosko AJ, et al. Intraoperative fluorescent angiography predicts pharyngocutaneous fistula after salvage laryngectomy. Ann Surg Oncol. 2019. https://doi.org/10.1245/s10 434-019-07262-6.

4. Alander JT, Kaartinen I, Laakso A, et al. A review of indocyanine green fluorescent imaging in surgery. Int J Biomed Imaging. 2012;2012:940585.

5. Moyer HR, Losken A. Predicting mastectomy skin flap necrosis with indocyanine green angiography: the gray area defined. Plast Reconstr Surg. 2012;129(5):1043-8.

6. Colavita PD, Wormer BA, Belyansky I, et al. Intraoperative indocyanine green fluorescence angiography to predict wound complications in complex ventral hernia repair. Hernia 2016;20:139-49.

7. Holm C, Tegeler J, Mayr M, et al. Monitoring free flaps using laser-induced fluorescence of indocyanine green: a preliminary experience. Microsurgery 2002;22(7):278-87.

8. Jafari MD, Wexner SD, Martz JE, et al. Perfusion assessment in laparoscopic left-sided/anterior resection (PILLAR II): a multiinstitutional study. J Am Coll Surg. 2015;220(1):82-92.

Publisher's Note Springer Nature remains neutral with regard to jurisdictional claims in published maps and institutional affiliations. 